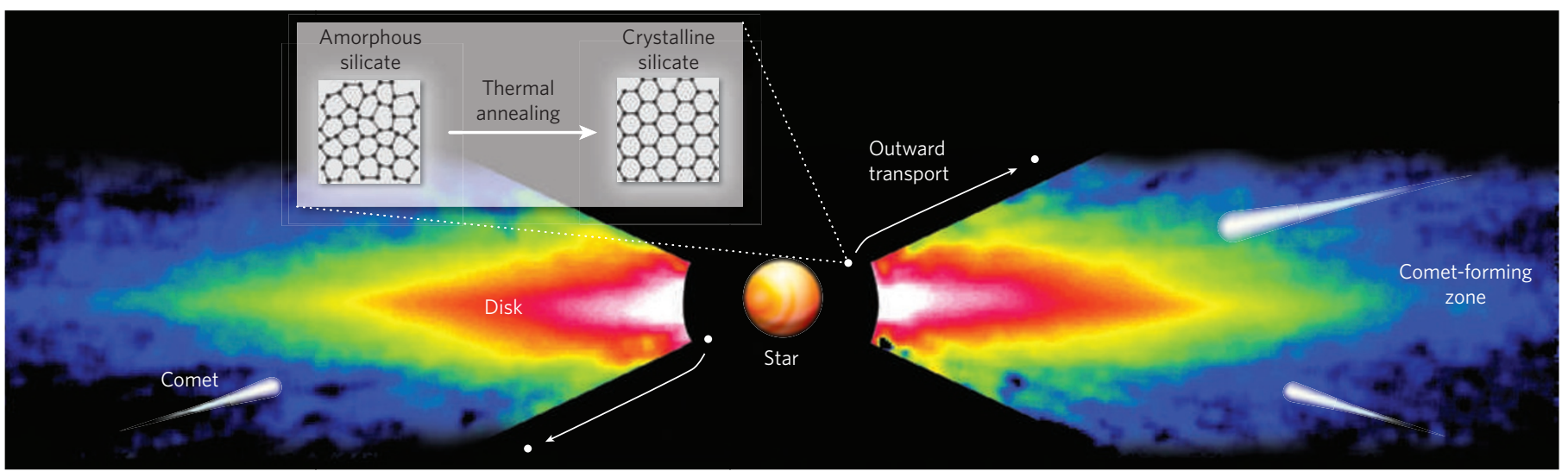

Figure 1 | Origin and transport of silicate crystals in planet-forming dust disks. According to the latest findings ${ }^{4,5}$, crystalline silicates are produced in the hot, inner regions of a star's disk by thermal annealing of amorphous silicates, and are subsequently transported to the cold, outer comet-forming regions, where they settle towards the disk's mid-plane and are incorporated into comets. In this artist's representation, the structures of the star, comets and silicates are overlaid on a (false-colour) visible-light Hubble Space Telescope view of the star Beta Pictoris's dust disk, which is seen tilted almost edge-on from the telescope's vantage point. The disk extends to more than $1,000 \mathrm{AU}$ from the star, with an inner dust-free hole of a few $\mathrm{AU}$ (presumably created by planets) ${ }^{15}$. The dark, circle-shaped region around the star is the result of light - originating from the star - being blocked by the coronagraph on Hubble. in the dust samples collected from comet $81 \mathrm{P} /$ Wild 2 by the Stardust spacecraft ${ }^{6}$. Their presence in other comets has also been revealed by infrared (IR) spectral signatures ${ }^{7}$ : the IR spectra display sharp emission features at several specific wavelengths that are characteristic of crystalline silicates. These distinct emission features are also seen in protoplanetary disks around young stars ${ }^{8}$, suggesting a similar origin for crystalline silicates in comets and in these dust disks.

So where did the observed silicate crystals in comets come from? Apparently, they were not inherited from the interstellar medium, simply because interstellar silicates are predominantly amorphous 9 . They clearly did not form in cometary nuclei, which are believed to be assembled at temperatures below $30 \mathrm{~K}$ (ref. 2), or in the cold, outer regions of the solar nebula, where comets were accreted about 4.5 billion years ago and where materials have never experienced temperatures higher than $100 \mathrm{~K}$. The crystallization of the original, amorphous silicates through thermal annealing requires temperatures of at least $\sim 1,000 \mathrm{~K}$ (ref. 10), in contradiction with the scenario in which comets were formed and stored in cold environments ${ }^{2}$.

The standard speculation has been that the volatile ices and crystalline silicates found in comets are of different origins. Whereas volatile ices may be pristine interstellar material surviving from the time the Solar System formed, crystalline silicates can originate from amorphous silicates that were transformed to crystalline form by thermal annealing in the hot, inner solar nebula, and were then transported outwards and incorporated into comets (Fig. 1).

However, it is Ábrahám et al. ${ }^{4}$ who provide the first concrete observational evidence for thermal annealing of amorphous silicates. They present mid-IR spectra, in the 5.2-37-micrometre wavelength range, of the star EX Lupi, obtained at two epochs separated by an interval of about 3 years. EX Lupi is a prototypical young Sun-like eruptive star that undergoes large, repetitive outbursts. The firstepoch spectrum, obtained when EX Lupi was in a quiescent phase, displays a broad, smooth 9.7- $\mu \mathrm{m}$ emission band, a telltale signature of amorphous silicates. By contrast, the secondepoch spectrum, acquired when the star was in the middle of an outburst, exhibits several sharp peaks characteristic of crystalline silicates superimposed on the broad, 9.7- $\mu \mathrm{m}$ band of amorphous silicates. These features are similar to those observed in comet spectra.

Ábrahám et al. ${ }^{4}$ interpret the observations as ongoing crystal formation: crystalline silicates are produced by thermal annealing in the surface layer of the star's inner disk (about 0.5 AU from the star) by heat from the outburst, which increases the visual brightness of the star by a factor of about 100. Alternative explanations for the observed spectral peaks, such as illumination of existing crystals residing in outer disk areas or the stirring up of crystals from the disk's mid-plane, are ruled out by modelling.

So the question that naturally arises is how these newly produced silicate crystals are carried outwards from the inner, crystalformation zone to the cold, comet-forming zone to be incorporated into comets? Several mechanisms have been suggested, including turbulent mixing ${ }^{11}$ of dust grains in the mid-plane of the solar nebula and the ' $\mathrm{X}$-wind' model, in which dust grains are ballistically launched above the disk's mid-plane and transported

\title{
MICROBIOLOGY
}

\section{Signals for change}

The strategy that the protozoan parasite

Trypanosoma brucei - which causes fatal disease in humans and cattle - uses to evade its host's immune defences is the subject of a News \& Views article on page 172. Elsewhere in this issue, Dean et al. provide further molecular insights into the workings of this pathogen (pictured, with red blood cell). They focus on the cell-differentiation events associated with its transmission between host and vector (S. Dean et al. Nature 459, 213-217; 2009).

In response to the metabolites citrate or cis-aconitate, trypanosomes differentiate from a non-dividing, stumpy form - the form thought to be taken up by the parasite's tsetse fly vector from the mammalian host's bloodstream - to a dividing form found in the fly's midgut.

The parasite becomes sensitive to the metabolite signals through exposure to low temperatures, which the fly often experiences while feeding at dusk or dawn.

But the surface molecules responsible for transmitting the signals to the microbe have remained elusive.

Dean and colleagues find that trypanosomes sense citrate through the PAD family of cell-surface transporter proteins. Only the stumpy form seems to express

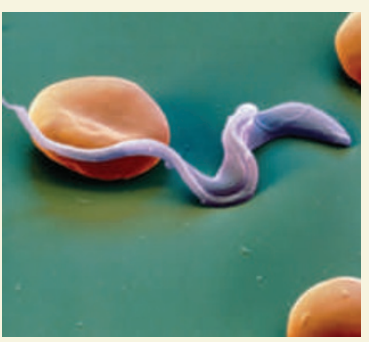

these proteins, establishing it as the competent stage in the parasite's life cycle for transmission from its mammalian host to the fly.

The authors also find that reducing PAD expression decreases citrate-induced trypanosome differentiation. PAD proteins could therefore potentially be used as molecular markers when screening for compounds that promote transition to the non-dividing, and so less-virulent, stumpy form. Sadaf Shadan 